\title{
Weak Estimates of Singular Integrals with Variable Kernel and Fractional Differentiation on Morrey-Herz Spaces
}

\author{
Yanqi Yang and Shuangping Tao \\ College of Mathematics and Statistics, Northwest Normal University, Lanzhou, Gansu 730070, China \\ Correspondence should be addressed to Shuangping Tao; taosp@nwnu.edu.cn
}

Received 9 October 2017; Accepted 12 December 2017; Published 31 December 2017

Academic Editor: Henryk Hudzik

Copyright (c) 2017 Yanqi Yang and Shuangping Tao. This is an open access article distributed under the Creative Commons Attribution License, which permits unrestricted use, distribution, and reproduction in any medium, provided the original work is properly cited.

Let $T$ be the singular integral operator with variable kernel defined by $T f(x)=$ p.v. $\int_{\mathbb{R}^{n}}\left(\Omega(x, x-y) /|x-y|^{n}\right) f(y) \mathrm{d} y$ and let $D^{\gamma}(0 \leq \gamma \leq 1)$ be the fractional differentiation operator. Let $T^{*}$ and $T^{\sharp}$ be the adjoint of $T$ and the pseudoadjoint of $T$, respectively. In this paper, the authors prove that $T D^{\gamma}-D^{\gamma} T$ and $\left(T^{*}-T^{\sharp}\right) D^{\gamma}$ are bounded, respectively, from Morrey-Herz spaces $M \dot{K}_{p, 1}^{\alpha, \lambda}\left(\mathbb{R}^{n}\right)$ to the weak Morrey-Herz spaces $W M \dot{K}_{p, 1}^{\alpha, \lambda}\left(\mathbb{R}^{n}\right)$ by using the spherical harmonic decomposition. Furthermore, several norm inequalities for the product $T_{1} T_{2}$ and the pseudoproduct $T_{1} \circ T_{2}$ are also given.

\section{Introduction}

Denote $\mathbb{S}^{n-1}$ to be the unit sphere in $\mathbb{R}^{n}(n \geq 2)$ with normalized Lebesgue measure $\mathrm{d} \sigma$. The singular integral operator with variable kernel is defined by

$$
T f(x)=\text { p.v. } \int_{\mathbb{R}^{n}} \frac{\Omega(x, x-y)}{|x-y|^{n}} f(y) \mathrm{d} y,
$$

where $\Omega(x, z)$ satisfies the following conditions:

$$
\begin{gathered}
\Omega(x, \lambda z)=\Omega(x, z), \\
\quad \text { for any } x, z \in \mathbb{R}^{n}, \lambda>0, \\
\int_{S^{n-1}} \Omega\left(x, z^{\prime}\right) \mathrm{d} \sigma\left(z^{\prime}\right)=0, \quad \text { for any } x \in \mathbb{R}^{n} .
\end{gathered}
$$

As we all know, the singular integrals with variable kernel played an important role in the theory of nondivergent elliptic equations with discontinuous coefficients (see $[1,2])$. Some properties for various of the singular integrals with variable kernel have been obtained by authors; for example, see [3-6] and their references. In the Mihlin conditions, Calderón and Zygmund proved the boundedness of $T$ on the $L^{2}\left(\mathbb{R}^{n}\right)$ (see [7]).
Let $0 \leq \gamma \leq 1$. For tempered distributions $f \in$ $\delta^{\prime}\left(\mathbb{R}^{n}\right)(n=1,2, \ldots)$, the fractional differentiation operators $D^{\gamma}$ defined by $\widehat{D^{\gamma} f}(\xi)=|\xi|^{\gamma} \widehat{f}(\xi)$; that is, $D^{\gamma} f(x)=$ $\left(|\xi|^{\gamma} \widehat{f}(\xi)\right)^{\vee}(x)$.

Let $I_{\gamma}$ be the Riesz potential operator of order $\gamma$ defined on the space of tempered distributions modulo polynomials by setting $\widehat{I_{\gamma} f}(\xi)=|\xi|^{-\gamma} \widehat{f}(\xi)$. It is easy to see that a locally integrable function $b \in I_{\gamma}(\mathrm{BMO})\left(\mathbb{R}^{n}\right)$ if and only if $D^{\gamma} b \in$ $\mathrm{BMO}\left(\mathbb{R}^{n}\right)$. Strichartz (see $[8]$ ) showed that $I_{\gamma}(\mathrm{BMO})\left(\mathbb{R}^{n}\right)$ is a space of functions modulo constants which is properly contained in $\operatorname{Lip}_{\gamma}\left(\mathbb{R}^{n}\right)$, where $\gamma \in(0,1)$.

Denote $\mathscr{H}_{m}$ to be the space of spherical harmonical homogeneous polynomials of degree $m$. Let $\operatorname{dim} \mathscr{H}_{m}=d_{m}$ and $\left\{Y_{m, j}\right\}_{j=1}^{d_{m}}$ be an orthonormal system of $\mathscr{H}_{m}$. It is well known that $\left\{Y_{m, j}\right\}_{j=1}^{d_{m}}, m=0,1, \ldots$, is a complete orthonormal system in $L^{2}\left(S^{n-1}\right)$ (see [9]). Let us expand the function $\Omega\left(x, z^{\prime}\right)$ in spherical harmonics

$$
\Omega\left(x, z^{\prime}\right)=\sum_{m \geq 0} \sum_{j=1}^{d_{m}} a_{m, j}(x) Y_{m, j}\left(z^{\prime}\right),
$$


where

$$
a_{m, j}(x)=\int_{S^{n-1}} \Omega\left(x, z^{\prime}\right) \overline{Y_{m, j}\left(z^{\prime}\right)} \mathrm{d} \sigma\left(z^{\prime}\right) .
$$

If $\int_{S^{n-1}} \Omega\left(x, z^{\prime}\right) \mathrm{d} \sigma\left(z^{\prime}\right)=0$, then $a_{0, j}=0$ for any $x \in \mathbb{R}^{n}$. Let

$$
T_{m, j} f(x)=\left(\frac{Y_{m, j}}{|\cdot|^{n}} * f\right)(x) .
$$

Then $T$, defined in (1), can be written as

$$
T f(x)=\sum_{m \geq 1} \sum_{j=1}^{d_{m}} a_{m, j}(x) T_{m, j} f(x) .
$$

Let $T^{*}$ and $T^{\sharp}$ be the adjoint of $T$ and the pseudoadjoint of $T$, respectively, defined by

$$
\begin{aligned}
& T^{*} f(x)=\sum_{m=1}^{\infty} \sum_{j=1}^{d_{m}}(-1)^{m} T_{m, j}\left(\bar{a}_{m, j} f\right)(x), \\
& T^{\sharp} f(x)=\sum_{m=1}^{\infty} \sum_{j=1}^{d_{m}}(-1)^{m} \bar{a}_{m, j}(x) T_{m, j} f(x) .
\end{aligned}
$$

Let us give some necessary notations. In the following, unless otherwise stated, for a $\mu$-measurable set $E, \chi_{E}$ denotes its characteristic function. We use the symbol $A \leqslant B$ to denote that there exists a positive constant $C$ such that $A \leq C B$. For any index $p \in(1, \infty)$, we denote by $p^{\prime}$ its conjugate index; that is, $1 / p+1 / p^{\prime}=1$.

Let $T_{1}$ and $T_{2}$ be the operators defined in (1) which are differentiated by their kernels $\Omega_{1}(x, y)$ and $\Omega_{2}(x, y)$. Let $T_{1} T_{2}, T_{1} \circ T_{2}$ denote the product and pseudoproduct of $T_{1}$ and $T_{2}$, respectively. In [7], Calderón and Zygmund found that these operators are closely related to the second order linear elliptic equations with variable coefficients and established the following boundedness of the operators $T_{1}^{*}, T_{1}^{\sharp}, T_{1} T_{2}$, $T_{1} \circ T_{2}$, and $D$ on $L^{p}\left(\mathbb{R}^{n}\right)(1<p<\infty)$.

Theorem A (see [7]). Let $1<p<\infty, \Omega_{1}(x, y), \Omega_{2}(x, y) \in$ $C^{\beta}\left(C^{\infty}\right), \beta>1$ satisfy (2) and (3). Then

(1) $\left\|\left(T_{1} D-D T_{1}\right) f\right\|_{L^{p}} \lesssim\|f\|_{L^{p}}$;

(2) $\left\|\left(T_{1}^{*}-T_{1}^{\sharp}\right) D f\right\|_{L^{p}} \lesssim\|f\|_{L^{p}}$;

(3) $\left\|\left(T_{1} \circ T_{2}-T_{1} T_{2}\right) D f\right\|_{L^{p}} \lesssim\|f\|_{L^{p}}$.

In 2015, Chen and Zhu proved that Theorem A was also true on Weighted Lebesgue space and Morrey space (see [10]). In 2016, Tao and Yang obtained the boundedness of those operators on the weighted Morrey-Herz spaces (see [6]). A natural question is whether these operators also have boundedness on the weak Morrey-Herz spaces. The answer is affirmative. The main purpose of this paper is to generalize the above results to the cases of weak Morrey-Herz spaces $W M \dot{K}_{p, 1}^{\alpha, \lambda}\left(\mathbb{R}^{n}\right)$ (see Definition 7 in the next section).

Our main results are stated as follows.
Theorem 1. Let $0<\gamma<1, \lambda-1<\alpha<\lambda$, and $0<p<\infty$. Assume that $T$ is defined by (1) and $\Omega(x, y)$, which satisfies (2) and (3), meets the following condition:

$$
\max _{|j| \leq 2 n}\left\|D_{x}^{\gamma}\left(\frac{\partial^{j}}{\partial y^{j}}\right) \Omega(x, y)\right\|_{L^{\infty}\left(\mathbb{R}^{n} \times S^{n-1}\right)}<\infty .
$$

Then one has

$$
\begin{aligned}
& \text { (1) }\left\|\left(T D^{\gamma}-D^{\gamma} T\right) f\right\|_{W M \dot{K}_{p, 1}^{\alpha, \lambda}\left(\mathbb{R}^{n}\right)} \lesssim\|f\|_{M \dot{K}_{p, 1}^{\alpha, \lambda}\left(\mathbb{R}^{n}\right)} ; \\
& \text { (2) }\left\|\left(T^{*}-T^{\sharp}\right) D^{\gamma} f\right\|_{W M \dot{K}_{p, 1}^{\alpha, \lambda}\left(\mathbb{R}^{n}\right)} \lesssim\|f\|_{M \dot{K}_{p, 1}^{\alpha, \lambda}\left(\mathbb{R}^{n}\right)}
\end{aligned}
$$

Theorem 2. Let $0<\gamma<1, \lambda-1<\alpha<\lambda$, and $0<p<$ $\infty$. Suppose that $\Omega_{1}(x, y)$ and $\Omega_{2}(x, y)$ satisfy (2) and (3). If $\Omega_{2}(x, y)$ satisfies (9) and $\Omega_{1}(x, y)$ satisfies

$$
\max _{|j| \leq 2 n}\left\|\left(\frac{\partial^{j}}{\partial y^{j}}\right) \Omega_{1}(x, y)\right\|_{L^{\infty}\left(\mathbb{R}^{n} \times S^{n-1}\right)}<\infty,
$$

then one has

$$
\left\|\left(T_{1} \circ T_{2}-T_{1} T_{2}\right) D^{\gamma} f\right\|_{W M \dot{K}_{p, 1}^{\alpha, \lambda}\left(\mathbb{R}^{n}\right)} \leqslant\|f\|_{M \dot{K}_{p, 1}^{\alpha, \lambda}\left(\mathbb{R}^{n}\right)} .
$$

Furthermore, we also consider the cases $\gamma=0$ and $\gamma=1$. As we all know, $D$ is the square root of Laplacian operator and $D^{0}$ is the identity operator $\mathscr{I}$. In this case, we obtain the following results.

Theorem 3. Let $\lambda-1<\alpha<\lambda$ and $0<p<\infty$. Suppose that $\Omega_{i}(x, y)(i=1,2)$ satisfies (2), (3), and (10). Then one has
(1) $\left\|\left(T_{1} \mathscr{I}-\mathscr{I} T_{1}\right) f\right\|_{W M \dot{K}_{p, 1}^{\alpha, \lambda}\left(\mathbb{R}^{n}\right)} \lesssim\|f\|_{M \dot{K}_{p, 1}^{\alpha, \lambda}\left(\mathbb{R}^{n}\right)} ;$
(2) $\left\|\left(T_{1}^{*}-T_{2}^{\sharp}\right) \mathscr{I} f\right\|_{W M \dot{K}_{p, 1}^{\alpha, \lambda}\left(\mathbb{R}^{n}\right)} \leqslant\|f\|_{M \dot{K}_{p, 1}^{\alpha, \lambda}\left(\mathbb{R}^{n}\right)}$;
(3) $\left\|\left(T_{1} \circ T_{2}-T_{1} T_{2}\right) \mathscr{I} f\right\|_{W M \dot{K}_{p, 1}^{\alpha, \lambda}\left(\mathbb{R}^{n}\right)} \lesssim\|f\|_{M \dot{K}_{p, 1}^{\alpha, \lambda}\left(\mathbb{R}^{n}\right)}$.

Theorem 4. Let $\lambda-1<\alpha<\lambda$ and $0<p<\infty$. Suppose that $\Omega(x, y)$ satisfies (2), (3), and

$$
\max _{|j| \leq 2 n}\left\|\nabla_{x}\left(\frac{\partial^{j}}{\partial y^{j}}\right) \Omega(x, y)\right\|_{L^{\infty}\left(\mathbb{R}^{n} \times S^{n-1}\right)}<\infty .
$$

Then one has

$$
\begin{aligned}
& \text { (1) }\|(T D-D T) f\|_{W M \dot{K}_{p, 1}^{\alpha, \lambda}\left(\mathbb{R}^{n}\right)} \\
& \text { (2) }\left\|\left(T^{*}-T^{\sharp}\right) D f\right\|_{W M \dot{K}_{p, 1}^{\alpha, \lambda}\left(\mathbb{R}^{n}\right)} \leqslant\|f\|_{M \dot{K}_{p, 1}^{\alpha, \lambda}\left(\mathbb{R}^{n}\right)} ;
\end{aligned}
$$

Theorem 5. Let $\lambda-1<\alpha<\lambda$ and $0<p<\infty$. Suppose that $\Omega_{1}(x, y)$ and $\Omega_{2}(x, y)$ satisfy (2) and (3). If $\Omega_{1}(x, y)$ satisfies (10) and $\Omega_{2}(x, y)$ satisfies (12), then one has

$$
\left\|\left(T_{1} \circ T_{2}-T_{1} T_{2}\right) D f\right\|_{W M \dot{K}_{p, 1}^{\alpha, \lambda}\left(\mathbb{R}^{n}\right)} \leqslant\|f\|_{M \dot{K}_{p, 1}^{\alpha, \lambda}\left(\mathbb{R}^{n}\right)} .
$$

\section{Preliminaries and Main Lemmas}

In this section, we shall recall the definitions of the homogeneous Morrey-Herz spaces and weak Morrey-Herz spaces. 
Furthermore, the weak estimates of $T_{m, j}$ defined by (6) and a class of Calderón-Zygmund operators will be established on Morrey-Herz spaces.

The well-known Morrey spaces, introduced originally by Morrey [11] in relation to the study of partial differential equations, were widely investigated during last decades, including the study of classical operators of harmonic analysis in various generalizations of these spaces. Morrey-type spaces appeared to be quite useful in the study of the local behavior of the solutions of partial differential equations, a priori estimates, and other topics. They are also widely used in applications to regularity properties of solutions to PDE including the study of Navier-Stokes equations (see [12] and references therein). The ideas of Morrey [11] were further developed by Campanato [13]. A more systematic study of these (and even more general) spaces, we refer the readers to see [12, 14-21]. In 1964, Beurling [22] first introduced some fundamental forms of Herz spaces to study convolution algebras. Later Herz [23] gave versions of the spaces defined below in a slightly different setting. Since then, the theory of Herz spaces has been significantly developed, and these spaces have turned out to be quite useful in harmonic analysis. For instance, they were used by Baernstein and Sawyer [24] to characterize the multipliers on the classical Hardy spaces and used by Lu and Yang [25] in the study of partial differential equations. More results and further details can be found in [26-28]. On the basis of above available results, the theory of the homogeneous Morrey-Herz spaces goes back to Lu-Xu [29] who considered the boundedness of a class of sublinear operators; also see $[6,30,31]$ for more further results.

Next we give the following notation. For each $k \in \mathbb{Z}$, we denote $B_{k}=\left\{x \in \mathbb{R}^{n}:|x| \leq 2^{k}\right\}$ and $A_{k}=B_{k}-B_{k-1}$, $\chi_{k}=\chi_{A_{k}}(x)$.

Definition 6 (see [29]). Let $\alpha \in \mathbb{R}^{n}, 0<p \leq \infty, 0<$ $q<\infty$, and $\lambda \geq 0$. The homogeneous Morrey-Herz spaces $M \dot{K}_{p, q}^{\alpha, \lambda}\left(\mathbb{R}^{n}\right)$ are defined by

$$
\begin{aligned}
& M \dot{K}_{p, q}^{\alpha, \lambda}\left(\mathbb{R}^{n}\right) \\
& \quad=\left\{f \in L_{\text {loc }}^{q}\left(\mathbb{R}^{n} \backslash 0\right),\|f\|_{M \dot{K}_{p, q}^{\alpha, \lambda}\left(\mathbb{R}^{n}\right)}<\infty\right\},
\end{aligned}
$$

where

$$
\|f\|_{M \dot{K}_{p, q}^{\alpha, \lambda}\left(\mathbb{R}^{n}\right)}=\sup _{k_{0} \in \mathbb{Z}} 2^{-k_{0} \lambda}\left(\sum_{k=-\infty}^{k_{0}} 2^{k \alpha p}\left\|f \chi_{k}\right\|_{L^{q}\left(\mathbb{R}^{n}\right)}^{p}\right)^{1 / p},
$$

and the usual modifications should be made when $p=\infty$.

In what follows, for any $k \in \mathbb{Z}$ and $\gamma>0$, let $m_{k}(\gamma, f)=$ $\left|\left\{x \in A_{k}:|f(x)|>\gamma\right\}\right|$.

Definition 7 (see [29]). Let $\alpha \in \mathbb{R}^{n}, 0<p \leq \infty, 0<q<\infty$, and $\lambda \geq 0$. A measurable function $f$ is said to belong to the homogeneous weak Morrey-Herz spaces $W M \dot{K}_{p, q}^{\alpha, \lambda}\left(\mathbb{R}^{n}\right)$, if

$$
\begin{array}{rl}
W & M \dot{K}_{p, q}^{\alpha, \lambda}\left(\mathbb{R}^{n}\right) \\
& =\left\{f \in L_{\mathrm{loc}}^{q}\left(\mathbb{R}^{n} \backslash 0\right),\|f\|_{W M \dot{K}_{p, q}^{\alpha, \lambda}\left(\mathbb{R}^{n}\right)}<\infty\right\},
\end{array}
$$

where

$$
\begin{aligned}
& \|f\|_{W M \dot{K}_{p, q}^{\alpha, \lambda}\left(\mathbb{R}^{n}\right)} \\
& \quad=\sup _{\gamma>0} \gamma \sup _{k_{0} \in \mathbb{Z}} 2^{-k_{0} \lambda}\left(\sum_{k=-\infty}^{k_{0}} 2^{k \alpha p} m_{k}(\gamma, f)^{p / q}\right)^{1 / p} \\
& \quad<\infty
\end{aligned}
$$

where the usual modifications are made when $p=\infty$.

Lemma 8 (see [32]). $(X, d, \mu)$ is said to be a homogeneous space. Let $0 \leq \lambda \leq \infty, 0 \leq l<1,1<q_{1}<1 / l, 1 / q_{2}=1 / q_{1}-l$, $\lambda-1 / q_{2}<\alpha<\lambda+1-1 / q_{1}$, and $0<p_{1} \leq p_{2}<\infty$, if a sublinear operator $T_{l}$ meets the following requirements:

$$
\left|T_{l}(f(x))\right| \leq C \int_{X} \frac{|f(y)|}{\mu\left(B\left(x_{0}, d(x, y)\right)\right)^{1-l}} \mathrm{~d} \mu(y),
$$

$$
x \notin \operatorname{supp} f \text {. }
$$

And $T_{l}$ is bounded from $L^{q_{1}}(X)$ to $L^{q_{2}, \infty}(X)$, and then $T_{l}$ is a bounded operator from $M \dot{K}_{p_{1}, q_{1}}^{\alpha, \lambda}(X)$ to $W M \dot{K}_{p_{2}, q_{2}}^{\alpha, \lambda}(X)$. When $q_{1}=1$, as can be seen from the proof process, the above conclusion is still valid.

Lemma 9. Let $T_{m, j}$ be defined by (6). Then $T_{m, j}$ is weak $(1,1)$, and

$$
\left|\left\{x \in \mathbb{R}^{n}:\left|T_{m, j} f\right|>\lambda\right\}\right| \lesssim m^{n / 2} \frac{1}{\lambda}\|f\|_{1} .
$$

Proof. Let $T_{m, j} f=Y_{m, j} /|\cdot|^{n} * f$ and $\left|Y_{m, j}\right| \lesssim m^{(n-2) / 2}$ ([7]). Fix $\lambda>0$ and $f \in L^{1}\left(\mathbb{R}^{n}\right)$. Form the Calderón-Zygmund decomposition of $f$ at height $\lambda$. Then for any $\lambda>0$, there exists a decomposition of $\mathbb{R}^{n}: \mathbb{R}^{n}=Q^{\lambda}+P^{\lambda}$ such that

(1) $|f(x)| \leq \lambda$ a.e. $x \in P^{\lambda}$,

(2) $Q^{\lambda}=\bigcup_{l=1}^{\infty} Q_{l}$, where $Q_{l}$ are finite overlapping, and

$$
\lambda<\frac{1}{\left|Q_{l}\right|} \int_{Q_{l}}|f(x)| \mathrm{d} x \leq 2^{n} \lambda .
$$

We now decompose $f$ as the sum of two functions, $g$ and $b$, defined by

$$
\begin{aligned}
& g(x)= \begin{cases}f(x), & x \notin Q^{\lambda} ; \\
\frac{1}{\left|Q_{l}\right|} \int_{Q_{l}} f(y) \mathrm{d} y, & x \in Q_{l},\end{cases} \\
& b(x)=\sum_{l} b_{l}(x),
\end{aligned}
$$

where

$$
b_{l}(x)=\left(f(x)-\frac{1}{\left|Q_{l}\right|} \int_{\mathrm{Q}_{l}} f(y) \mathrm{d} y\right) \chi_{\mathrm{Q}_{l}}(x) .
$$


Then $g(x) \leq 2^{n} \lambda$ almost everywhere, and $b_{l}$ is supported on $Q_{l}$ and has zero integral. Since $T_{m, j} f=T_{m, j} g+T_{m, j} b$,

$$
\begin{aligned}
\mid\{x \in & \left.\in \mathbb{R}^{n}:\left|T_{m, j} f(x)\right|>\lambda\right\} \mid \\
\leq & \left|\left\{x \in \mathbb{R}^{n}:\left|T_{m, j} g(x)\right|>\frac{\lambda}{2}\right\}\right| \\
& +\left|\left\{x \in \mathbb{R}^{n}:\left|T_{m, j} b(x)\right|>\frac{\lambda}{2}\right\}\right| .
\end{aligned}
$$

We estimate the first term, in view of the fact that $\left\|T_{m, j} f\right\|_{L^{p}\left(\mathbb{R}^{n}\right)} \lesssim m^{n / 2}\|f\|_{L^{p}\left(\mathbb{R}^{n}\right)}([10])$. Without loss of generality, for $p=2$, we have

$$
\begin{aligned}
& \left|\left\{x \in \mathbb{R}^{n}:\left|T_{m, j} g(x)\right|>\frac{\lambda}{2}\right\}\right| \\
& \leq\left(\frac{2}{\lambda}\right)^{2} \int_{\mathbb{R}^{n}}\left|T_{m, j} g(x)\right|^{2} \mathrm{~d} x \\
& \quad \leq m^{n / 2} \frac{4}{\lambda^{2}} \int_{\mathbb{R}^{n}} g(x)^{2} \mathrm{~d} x \leq m^{n / 2} \frac{2^{n+2}}{\lambda} \int_{\mathbb{R}^{n}}|g(x)| \mathrm{d} x \\
& \quad=m^{n / 2} \frac{2^{n+2}}{\lambda} \int_{\mathbb{R}^{n}}|f(x)| \mathrm{d} x .
\end{aligned}
$$

Let $2 Q_{l}$ be the cube with the same center as $Q_{l}$ and whose sides are twice as long, and let $\left(Q^{\lambda}\right)^{*}=\bigcup_{l} 2 Q_{l}$. Then we have $\left|\left(Q^{\lambda}\right)^{*}\right| \leq 2^{n}\left|Q^{\lambda}\right|$, and together with characteristic of left in (2), we can obtain

$$
\begin{aligned}
\left|Q_{\lambda}\right| & =\left|\bigcup_{l=1}^{\infty} Q_{l}\right| \leq \sum_{l=1}^{\infty}\left|Q_{l}\right| \leq \frac{1}{\lambda} \sum_{l=1}^{\infty} \int_{Q_{l}}|f(x)| \mathrm{d} x \\
& \leq \frac{1}{\lambda} \int_{Q^{\lambda}}|f(x)| \mathrm{d} x \leq \frac{1}{\lambda}\|f\|_{1} .
\end{aligned}
$$

Thus we have

$$
\begin{aligned}
& \left|\left\{x \in \mathbb{R}^{n}:\left|T_{m, j} b(x)\right|>\frac{\lambda}{2}\right\}\right| \\
& \quad \leq\left|\left(Q^{\lambda}\right)^{*}\right|+\left|\left\{x \notin\left(Q^{\lambda}\right)^{*}:\left|T_{m, j} b(x)>\frac{\lambda}{2}\right|\right\}\right| \\
& \quad \leq \frac{2^{n}}{\lambda}\|f\|_{1}+\frac{2}{\lambda} \int_{\mathbb{R}^{n} \backslash\left(Q^{\lambda}\right)^{*}}\left|T_{m, j} b(x)\right| \mathrm{d} x .
\end{aligned}
$$

Notice that $\left|T_{m, j} b(x)\right| \leq \sum_{l}\left|T_{m, j} b_{l}(x)\right|$ almost everywhere. Hence, to complete the proof of the weak $(1,1)$ inequality it will suffice to show that

$$
\sum_{l} \int_{\mathbb{R}^{n} \mid 2 Q_{l}}\left|T_{m, j} b_{l}(x)\right| \mathrm{d} x \leqslant\|f\|_{1} .
$$

For any $b_{l}, x \notin 2 Q_{l}$, the formula

$$
T_{m, j} b_{l}(x)=\int_{\mathrm{Q}_{l}} \frac{b_{l}(y) Y_{m, j}(x-y)}{|x-y|^{n}} \mathrm{~d} y
$$

is still valid. Denote the center of $Q_{l}$ by $z_{Q_{l}}$, and then we have

$$
\begin{aligned}
& \int_{\mathbb{R}^{n} \backslash 2 \mathrm{Q}_{l}}\left|T_{m, j} b_{l}(x)\right| \mathrm{d} x \\
& =\int_{\mathbb{R}^{n} \backslash 2 \mathrm{Q}_{l}} \int_{\mathrm{Q}_{l}}\left|\frac{Y_{m, j}(x-y) b_{l}(y)}{|x-y|^{n}} \mathrm{~d} y\right| \mathrm{d} x \\
& \leq \int_{\mathbb{R}^{n} \mid 2 \mathrm{Q}_{l}} \mid \int_{\mathrm{Q}_{l}} b_{l}(y) Y_{m, j}(x-y) \\
& \cdot\left(\frac{1}{|x-y|^{n}}-\frac{1}{\mid x-z_{\left.\mathrm{Q}_{l}\right|^{n}}}\right) \mathrm{d} y \mid \mathrm{d} x \\
& \quad \leqslant m^{n / 2} \int_{\mathrm{Q}_{l}}\left|b_{l}(y)\right| \\
& \cdot\left(\int_{\mathbb{R}^{n} \mid 2 \mathrm{Q}_{l}} \frac{\mid y-z_{\left.\mathrm{Q}_{l}\right|^{n}}}{|x-y|^{n}\left|x-z_{\mathrm{Q}_{l}}\right|^{n}} \mathrm{~d}\right) \mathrm{d} y \\
& \quad \leqslant m^{n / 2} \int_{\mathrm{Q}_{l}}\left|b_{l}(y)\right|\left(\int_{\mathbb{R}^{n} \mid 2 \mathrm{Q}_{l}} \frac{\left|x-z_{\mathrm{Q}_{l}}\right|^{2 n}}{\mid} \mathrm{d} x\right) \mathrm{d} y .
\end{aligned}
$$

Noticing that

$$
\begin{aligned}
\int_{\mathbb{R}^{n} \mid 2 \mathrm{Q}_{l}} \frac{\left|\mathrm{Q}_{l}\right|}{\left|x-z_{\mathrm{Q}_{l} \mid}\right|^{2 n}} \mathrm{~d} x & =\sum_{k=1}^{\infty} \int_{2^{k+1} \mathrm{Q}_{l} \mid 2^{k} \mathrm{Q}_{l}} \frac{\left|\mathrm{Q}_{l}\right|}{\left|x-z_{\mathrm{Q}_{l}}\right|^{2 n}} \mathrm{~d} x \\
& \leq \sum_{k=1}^{\infty} \frac{\left|2^{k+1} \mathrm{Q}_{l}\right|\left|\mathrm{Q}_{l}\right|}{\left[l\left(2^{k} \mathrm{Q}_{l}\right)\right]^{2 n}}=\sum_{k=1}^{\infty} 2^{-k n+n} \\
& <\infty,
\end{aligned}
$$

it follows that

$$
\begin{aligned}
& \sum_{l} \int_{\mathbb{R}^{n} \backslash 2 \mathrm{Q}_{l}}\left|T_{m, j} b_{l}(x)\right| \mathrm{d} x \lesssim m^{n / 2} \sum_{l} \int_{\mathrm{Q}_{l}}\left|b_{l}(y)\right| \mathrm{d} y \\
& \quad \leq m^{n / 2}\|f\|_{1} .
\end{aligned}
$$

Then for any $T_{m, j}$, it is clear that

$$
\left|\left\{x \in \mathbb{R}^{n}:\left|T_{m, j} b(x)\right|>\frac{\lambda}{2}\right\}\right| \lesssim m^{n / 2} \frac{1}{\lambda}\|f\|_{1} .
$$

Summing up the estimates above for $T_{m, j} g(x)$ and $T_{m, j} b(x)$, we finish the proof of Lemma 9.

Lemma 10. Let $0 \leq \lambda \leq \infty, \lambda-1<\alpha<\lambda$, and $0<p<\infty$. Let $T$ be a generalized Calderón-Zygmund operator, and then $T$ is bounded from $M \dot{K}_{p, 1}^{\alpha, \lambda}\left(\mathbb{R}^{n}\right)$ to $W M \dot{K}_{p, 1}^{\alpha, \lambda}\left(\mathbb{R}^{n}\right)$; namely,

$$
\|T f(x)\|_{W M \dot{K}_{p, 1}^{\alpha, \lambda}\left(\mathbb{R}^{n}\right)} \leqslant\|f(x)\|_{M \dot{K}_{p, 1}^{\alpha, \lambda}\left(\mathbb{R}^{n}\right)} .
$$

Proof. It is well known that $T$ is weak (1) (e.g., see [33]). Noticing that $T$ satisfying (18) with $l=0$, then we can obtain Lemma 10 by using Lemma 8 . 
Lemma 11. Let $0 \leq \lambda \leq \infty, \lambda-1<\alpha<\lambda$, and $0<$ $p<\infty ; T_{m, j}$ defined by (6) is bounded from $M \dot{K}_{p, 1}^{\alpha, \lambda}\left(\mathbb{R}^{n}\right)$ to $W M \dot{K}_{p, 1}^{\alpha, \lambda}\left(\mathbb{R}^{n}\right)$, and

$$
\left\|T_{m, j} f(x)\right\|_{W M \dot{K}_{p, 1}^{\alpha, \lambda}\left(\mathbb{R}^{n}\right)} \leqslant m^{n / 2}\|f(x)\|_{M \dot{K}_{p, 1}^{\alpha, \lambda}\left(\mathbb{R}^{n}\right)} .
$$

Proof. By applying the fact that $T_{m, j} f(x)=Y_{m, j} /|\cdot|^{n} * f(x)$ and $\left|Y_{m, j}\right| \leqslant m^{(n-2) / 2}([7])$, we can easily obtain

$$
\begin{aligned}
\left|T_{m, j} f(x)\right| & \leqslant m^{(n-2) / 2} \int_{\mathbb{R}^{n}} \frac{|f(y)|}{|x-y|^{n}} \mathrm{~d} y \\
& \leqslant m^{n / 2} \int_{\mathbb{R}^{n}} \frac{|f(y)|}{|x-y|^{n}} \mathrm{~d} y .
\end{aligned}
$$

Noticing that the interior integral above is meeting the condition in (18) for $l=0$ and $T_{m, j}$ is weak (1) on the basis of Lemma 9, it is not difficult to deduce that $T_{m, j}$ is bounded from $M \dot{K}_{p, 1}^{\alpha, \lambda}\left(\mathbb{R}^{n}\right)$ to $W M \dot{K}_{p, 1}^{\alpha, \lambda}\left(\mathbb{R}^{n}\right)$. Hence

$$
\left\|T_{m, j} f(x)\right\|_{W M \dot{K}_{p, 1}^{\alpha, \lambda}\left(\mathbb{R}^{n}\right)} \leqslant m^{n / 2}\|f(x)\|_{M \dot{K}_{p, 1}^{\alpha, \lambda}\left(\mathbb{R}^{n}\right)} .
$$

This completes the proof of Lemma 11.

Lemma 12. Let $t(x)$ be a homogeneous of degree $-n-1$ and locally integrable in $|x|>0$. Let $b \in \operatorname{Lip}\left(\mathbb{R}^{n}\right)$ and

$$
K f(x)=\lim _{\varepsilon \mapsto 0} \int_{|x-y|>\varepsilon} t(x)(b(x)-b(y)) f(y) \mathrm{d} y .
$$

If $t(x) \in \mathscr{C}^{1}\left(S^{n-1}\right)$ and $\int_{S^{n-1}} t(x) x_{j} \mathrm{~d} \sigma(x)=0, j=1, \ldots, n$, then, for $0 \leq \lambda \leq \infty, \lambda-1<\alpha<\lambda$, and $0<p<\infty$, one has

$$
\begin{aligned}
& \|K f\|_{W M \dot{K}_{p, 1}^{\alpha, \lambda}\left(\mathbb{R}^{n}\right)} \\
& \quad \leq\left(\|\nabla t\|_{L^{\infty}\left(S^{n-1}\right)}+\|t\|_{L^{\infty}\left(S^{n-1}\right)}\right)\|\nabla b\|_{L^{\infty}}\|f\|_{M \dot{K}_{p, 1}^{\alpha, \lambda}\left(\mathbb{R}^{n}\right)} .
\end{aligned}
$$

Proof. Let $k(x, y)=t(x-y)(b(x)-b(y))$. For all $x, x_{0}, y \in$ $\mathbb{R}^{n}$ with $\left|x-x_{0}\right| \leq 1 / 2|y-x|$, then $k$ satisfies the following inequalities:

$$
\begin{aligned}
& \left|k(x, y)-k\left(x_{0}, y\right)\right| \\
& \quad \leq\|\nabla t\|_{L^{\infty}\left(S^{n-1}\right)}\|\nabla b\|_{L^{\infty}}\left|x-x_{0}\right||y-x|^{-n-1}, \\
& |k(x, y)| \lesssim\|t\|_{L^{\infty}\left(S^{n-1}\right)}\|\nabla b\|_{L^{\infty}}|y-x|^{-n} .
\end{aligned}
$$

This, together with the boundedness of $K$ on $L^{2}\left(\mathbb{R}^{n}\right)$ (see [34]), tells us that $K$ is a generalized Calderón-Zygmund operator ([33]). Thus, by applying Lemma 10 , we see that $K$ is bounded from $M \dot{K}_{p, 1}^{\alpha, \lambda}\left(\mathbb{R}^{n}\right)$ to $W M \dot{K}_{p, 1}^{\alpha, \lambda}\left(\mathbb{R}^{n}\right)$ with bound $\left(\|\nabla t\|_{L^{\infty}\left(S^{n-1}\right)}+\|t\|_{L^{\infty}\left(S^{n-1}\right)}\right)\|\nabla b\|_{L^{\infty}}$ for $0 \leq \lambda \leq \infty, \lambda-1<\alpha<\lambda$, and $0<p<\infty$.

Therefore, the proof of Lemma 12 is finished.

Lemma 13. Let $b \in \operatorname{Lip}\left(\mathbb{R}^{n}\right)$ and $T$ be a singular operator which is defined by

$$
T f(x)=\lim _{\varepsilon \mapsto 0} \int_{|x-y|>\varepsilon} K(x-y) f(y) \mathrm{d} y,
$$

where $K(x) \in \mathscr{C}^{3}\left(S^{n-1}\right), \int_{S^{n-1}} K(x) \mathrm{d} \sigma(x)=0$, and $K(\lambda x)=$ $\lambda^{-n} K(x)$, for $x \in \mathbb{R}^{n} \backslash\{0\}, \lambda>0$, and then one has that, for $0 \leq \lambda \leq \infty, \lambda-1<\alpha<\lambda, 0<p<\infty$, and $f \in C_{0}^{\infty}$, the operator

$$
\begin{aligned}
& \left\|[b, T] \frac{\partial f}{\partial x_{j}}\right\|_{W M \dot{K}_{p, 1}^{\alpha, \lambda}\left(\mathbb{R}^{n}\right)} \\
& \quad \leq \max _{|\beta| \leq 2}\left\|\partial^{\beta} K\right\|_{L^{\infty}\left(S^{n-1}\right)}\|\nabla b\|_{L^{\infty}}\|f\|_{M \dot{K}_{p, 1}^{\alpha, \lambda}\left(\mathbb{R}^{n}\right)} .
\end{aligned}
$$

Proof. With an argument similar to that used in the proof of Lemma 5.2 in [10], it is not difficult to obtain Lemma 13. Thus, we omit the details here.

\section{Proofs of Theorems}

Proof of Theorem 1. Let

$$
\Omega(x, y)=\sum_{m \geq 1} \sum_{j=1}^{d_{m}} a_{m, j}(x) Y_{m, j}(y) .
$$

From [3], for any $x$, we can write the coefficients $a_{m, j}$ as

$$
\begin{aligned}
a_{m, j}(x)= & (-1)^{n} m^{-n}(m+n-2)^{-n} \\
& \cdot \int_{S^{n-1}} L_{y^{\prime}}^{n}\left(\Omega\left(x, y^{\prime}\right)\right) Y_{m, j}\left(y^{\prime}\right) \mathrm{d} \sigma\left(y^{\prime}\right),
\end{aligned}
$$

$$
m \geq 1,
$$

where $L(F)=|x|^{2} \Delta F(x)$.

We will firstly prove conclusion (1). Write

$$
\begin{aligned}
& \left(T D^{\gamma}-D^{\gamma} T\right) f \\
& =\sum_{m=1}^{\infty} \sum_{j=1}^{d_{m}}\left(a_{m, j} T_{m, j} D^{\gamma}-D^{\gamma} a_{m, j} T_{m, j}\right) f \\
& =\sum_{m=1}^{\infty} \sum_{j=1}^{d_{m}}\left(a_{m, j} D^{\gamma} T_{m, j}-D^{\gamma} a_{m, j} T_{m, j}\right) f \\
& =: \sum_{m=1}^{\infty} \sum_{j=1}^{d_{m}}\left[a_{m, j}, D^{\gamma}\right] T_{m, j} f .
\end{aligned}
$$

By (43), it follows that

$$
\begin{aligned}
& D^{\gamma} a_{m, j}(x)=(-1)^{n} m^{-n}(m+n-2)^{-n} \\
& \quad \cdot \int_{S^{n-1}} D_{x}^{\gamma} L_{y^{\prime}}^{n}\left(\Omega\left(x, y^{\prime}\right)\right) Y_{m, j}\left(y^{\prime}\right) \mathrm{d} \sigma\left(y^{\prime}\right),
\end{aligned}
$$

$m \geq 1$.

Then, we have by (9)

$$
\left\|D^{\gamma} a_{m, j}\right\|_{L^{\infty}} \lesssim m^{-2 n} .
$$


Moreover, by the fact that $\left[b, D^{\gamma}\right]$ is a generalized CalderónZygmund operator (see [35]), which is defined by

$$
\left[b, D^{\gamma}\right] f(x)=C(\gamma) \int_{\mathbb{R}^{n}} \frac{(b(x)-b(y))}{|x-y|^{n+\gamma}} f(y) \mathrm{d} y .
$$

Thus we can get that $\left[b, D^{\gamma}\right] f(x)$ is bounded from $M \dot{K}_{p, 1}^{\alpha, \lambda}\left(\mathbb{R}^{n}\right)$ to $W M \dot{K}_{p, 1}^{\alpha, \lambda}\left(\mathbb{R}^{n}\right)$ by applying Lemma 10; namely,

$$
\left\|\left[b, D^{\gamma}\right] f\right\|_{W M \dot{K}_{p, 1}^{\alpha, \lambda}\left(\mathbb{R}^{n}\right)} \leqslant\left\|D^{\gamma} b\right\|_{\mathrm{BMO}}\|f\|_{M \dot{K}_{p, 1}^{\alpha, \lambda}\left(\mathbb{R}^{n}\right)} .
$$

Then by $d_{m} \simeq m^{n-2}$ (see [36]), (46), (48), and Lemma 11, we have

$$
\begin{aligned}
& \left\|\left(T D^{\gamma}-D^{\gamma} T\right) f\right\|_{W M \dot{K}_{p, 1}^{\alpha, \lambda}\left(\mathbb{R}^{n}\right)} \\
& \leq \sum_{m=1}^{\infty} \sum_{j=1}^{d_{m}}\left\|\left[a_{m, j}, D^{\gamma}\right] T_{m, j} f\right\|_{W M \dot{K}_{p, 1}^{\alpha, \lambda}\left(\mathbb{R}^{n}\right)} \\
& \leq \sum_{m=1}^{\infty} \sum_{j=1}^{d_{m}}\left\|D^{\gamma} a_{m, j}\right\|_{B M O}\left\|T_{m, j} f\right\|_{W M \dot{K}_{p, 1}^{\alpha, \lambda}\left(\mathbb{R}^{n}\right)} \\
& \leq \sum_{m=1}^{\infty} \sum_{j=1}^{d_{m}} m^{n / 2}\left\|D^{\gamma} a_{m, j}\right\|_{L^{\infty}}\|f\|_{M \dot{K}_{p, 1}^{\alpha, \lambda}\left(\mathbb{R}^{n}\right)} \\
& \quad \leq \sum_{m=1}^{\infty} m^{n-2} m^{n / 2} m^{-2 n}\|f\|_{M \dot{K}_{p, 1}^{\alpha, \lambda}\left(\mathbb{R}^{n}\right)} \leq\|f\|_{M \dot{K}_{p, 1}^{\alpha, \lambda}\left(\mathbb{R}^{n}\right)} .
\end{aligned}
$$

Now let us turn to estimate (2). By applying the definition of $T^{\sharp}$ and $T^{*}$ we can deduce that

$$
\left(T^{\sharp}-T^{*}\right) D^{\gamma} f=\sum_{m=1}^{\infty} \sum_{j=1}^{d_{m}}(-1)^{m}\left[\bar{a}_{m, j}, T_{m, j}\right] D^{\gamma} f .
$$

In order to estimate $W M \dot{K}_{p, 1}^{\alpha, \lambda}\left(\mathbb{R}^{n}\right)$ norm of $\left(T^{*}-T^{\sharp}\right) D^{\gamma}$, we first consider $\left[b, T_{m, j}\right] D^{\gamma}$ for any fixed $b \in I_{\gamma}(\mathrm{BMO})$. Noting that $b(x)-b(y)=(b(x)-b(z))-(b(y)-b(z))$, for any $x, y, z \in$ $\mathbb{R}^{n}$, then we have

$$
\left[b, T_{m, j}\right] D^{\gamma} f=\left[b, D^{\gamma} T_{m, j}\right] f-T_{m, j}\left[b, D^{\gamma}\right] f .
$$

Thus, by (48) and Lemma 11, we get

$$
\begin{aligned}
& \left\|T_{m, j}\left[b, D^{\gamma}\right] f\right\|_{W M \dot{K}_{p, 1}^{\alpha, \lambda}\left(\mathbb{R}^{n}\right)} \\
& \quad \leq m^{n / 2}\left\|D^{\gamma} b\right\|_{\mathrm{BMO}}\|f\|_{M \dot{K}_{p, 1}^{\alpha, \lambda}\left(\mathbb{R}^{n}\right)} .
\end{aligned}
$$

Further, we estimate the $W M \dot{K}_{p, 1}^{\alpha, \lambda}\left(\mathbb{R}^{n}\right)$ norm of $\left[b, D^{\gamma} T_{m, j}\right]$. From the fact that $\left[b, D^{\gamma} T_{m, j}\right] f$ is a generalized CalderónZygmund operator with kernel (see [10])

$$
\left|k_{m, j}(x, y)\right| \lesssim m^{n / 2-1+\gamma}\left\|D^{\gamma} b\right\|_{\mathrm{BMO}} \frac{1}{|x-y|^{n}},
$$

then we get by Lemma 10

$$
\begin{aligned}
& \left\|\left[b, D^{\gamma} T_{m, j}\right] f\right\|_{W M \dot{K}_{p, 1}^{\alpha, \lambda}\left(\mathbb{R}^{n}\right)} \\
& \quad \leq m^{n / 2+\gamma}\left\|D^{\gamma} b\right\|_{\mathrm{BMO}}\|f\|_{M \dot{K}_{p, 1}^{\alpha, \lambda}\left(\mathbb{R}^{n}\right)} .
\end{aligned}
$$

Then, combining (52) with (54), we have

$$
\begin{aligned}
\left\|\left[b, T_{m, j}\right] D^{\gamma} f\right\|_{W M \dot{K}_{p, 1}^{\alpha, \lambda}\left(\mathbb{R}^{n}\right)} \\
\leq\left\|\left[b, D^{\gamma} T_{m, j}\right] f\right\|_{W M \dot{K}_{p, 1}^{\alpha, \lambda}\left(\mathbb{R}^{n}\right)} \\
+\left\|T_{m, j}\left[b, D^{\gamma}\right] f\right\|_{W M \dot{K}_{p, 1}^{\alpha, \lambda}\left(\mathbb{R}^{n}\right)} \\
\leq m^{n / 2+\gamma}\left\|D^{\gamma} b\right\|_{\text {BMO }}\|f\|_{M \dot{K}_{p, 1}^{\alpha, \lambda}\left(\mathbb{R}^{n}\right)} \\
\quad+m^{n / 2}\left\|D^{\gamma} b\right\|_{\text {BMO }}\|f\|_{M \dot{K}_{p, 1}^{\alpha, \lambda}\left(\mathbb{R}^{n}\right)} \\
\leq m^{n / 2+\gamma}\left\|D^{\gamma} b\right\|_{B M O}\|f\|_{M \dot{K}_{p, 1}^{\alpha, \lambda}\left(\mathbb{R}^{n}\right)} .
\end{aligned}
$$

By estimates (46), (50), and (55), we get

$$
\begin{aligned}
& \left\|\left(T^{\sharp}-T^{*}\right) D^{\gamma} f\right\|_{W M \dot{K}_{p, 1}^{\alpha, \lambda}\left(\mathbb{R}^{n}\right)} \\
& \leq \sum_{m=1}^{\infty} \sum_{j=1}^{d_{m}}\left\|\left[\bar{a}_{m, j}, T_{m, j}\right] D^{\gamma} f\right\|_{W M \dot{K}_{p, 1}^{\alpha, \lambda}\left(\mathbb{R}^{n}\right)} \\
& \leq \sum_{m=1}^{\infty} \sum_{j=1}^{d_{m}} m^{n / 2+\gamma}\left\|D^{\gamma} \bar{a}_{m, j}\right\|_{B M O}\|f\|_{M \dot{K}_{p, 1}^{\alpha, \lambda}\left(\mathbb{R}^{n}\right)} \\
& \leq \sum_{m=1}^{\infty} \sum_{j=1}^{d_{m}} m^{n / 2+\gamma}\left\|D^{\gamma} \bar{a}_{m, j}\right\|_{L^{\infty}}\|f\|_{M \dot{K}_{p, 1}^{\alpha, \lambda}\left(\mathbb{R}^{n}\right)} \\
& \leq \sum_{m=1}^{\infty} m^{n-2} m^{n / 2+\gamma} m^{-2 n}\|f\|_{M \dot{K}_{p, 1}^{\alpha, \lambda}\left(\mathbb{R}^{n}\right)} \\
& \leq\|f\|_{M \dot{K}_{p, 1}^{\alpha, \lambda}\left(\mathbb{R}^{n}\right)} \cdot
\end{aligned}
$$

Thus we finish the proof of Theorem 1 .

Proof of Theorem 2. Let

$$
\begin{aligned}
& T_{1} f(x)=\int_{\mathbb{R}^{n}} \frac{\Omega_{1}(x, x-y)}{|x-y|^{n}} f(y) \mathrm{d} y, \\
& T_{2} f(x)=\int_{\mathbb{R}^{n}} \frac{\Omega_{2}(x, x-y)}{|x-y|^{n}} f(y) \mathrm{d} y .
\end{aligned}
$$

Write

$$
\begin{aligned}
& \Omega_{1}(x, y)=\sum_{m \geq 1} \sum_{j=1}^{d_{m}} a_{m, j}(x) Y_{m, j}(y), \\
& \Omega_{2}(x, y)=\sum_{\lambda \geq 1} \sum_{\mu=1}^{d_{\lambda}} b_{\lambda, \mu}(x) Y_{\lambda, \mu}(y),
\end{aligned}
$$


where

$$
\begin{aligned}
& a_{m, j}(x)=\int_{S^{n-1}} \Omega_{1}\left(x, z^{\prime}\right) \overline{Y_{m, j}\left(z^{\prime}\right)} \mathrm{d} \sigma\left(z^{\prime}\right), \\
& b_{\lambda, \mu}(x)=\int_{S^{n-1}} \Omega_{2}\left(x, z^{\prime}\right) \overline{Y_{\lambda, \mu}\left(z^{\prime}\right)} \mathrm{d} \sigma\left(z^{\prime}\right) .
\end{aligned}
$$

For any $x \in \mathbb{R}^{n}$, with a similar argument used in the proof of Theorem 1 in terms of (9) and (10), we can obtain that

$$
\begin{aligned}
\left\|a_{m, j}\right\|_{L^{\infty}} & \leqslant m^{-2 n}, \\
\left\|D^{\gamma} b_{\lambda, \mu}\right\|_{L^{\infty}} & \leqslant m^{-2 n} .
\end{aligned}
$$

Let $T_{m, j} f(x)=Y_{m, j} /|\cdot|^{n} * f(x)$ and $T_{\lambda, \mu} f(x)=Y_{\lambda, \mu} / \mid$. $I^{n} * f(x)$. Since $\Omega_{1}(x, y)$ and $\Omega_{2}(x, y)$ satisfy (3), then we get

$$
\begin{aligned}
& T_{1} f(x)=\sum_{m \geq 1} \sum_{j=1}^{d_{m}} a_{m, j}(x) T_{m, j} f(x), \\
& T_{2} f(x)=\sum_{\lambda \geq 1} \sum_{\mu=1}^{d_{\lambda}} b_{\lambda, \mu}(x) T_{\lambda, \mu} f(x) .
\end{aligned}
$$

Write ([10])

$$
\begin{aligned}
& \left(T_{1} \circ T_{2}\right) f(x) \\
& =\sum_{m=1}^{\infty} \sum_{j=1}^{d_{m}} \sum_{\lambda=1}^{\infty} \sum_{\mu=1}^{d_{\lambda}} a_{m, j}(x) b_{\lambda, \mu}(x)\left(T_{m, j} T_{\lambda, \mu} f\right)(x), \\
& \left(T_{1} T_{2}\right) f(x)=\sum_{m=1}^{\infty} \sum_{j=1}^{d_{m}} \sum_{\lambda=1}^{\infty} \sum_{\mu=1}^{d_{\lambda}} a_{m, j} T_{m, j}\left(b_{\lambda, \mu} T_{\lambda, \mu} f\right)(x) .
\end{aligned}
$$

Then

$$
\begin{aligned}
& \left(T_{1} \circ T_{2}-T_{1} T_{2}\right) D^{\gamma} f=\sum_{m=1}^{\infty} \sum_{j=1}^{d_{m}} \sum_{\lambda=1}^{\infty} \sum_{\mu=1}^{d_{\lambda}} a_{m, j} \\
& \cdot\left(b_{\lambda, \mu}(x) T_{m, j}-T_{m, j} b_{\lambda, \mu}(x)\right) T_{\lambda, \mu} D^{\gamma} f \\
& =\sum_{m=1}^{\infty} \sum_{j=1}^{d_{m}} \sum_{\lambda=1}^{\infty} \sum_{\mu=1}^{d_{\lambda}} a_{m, j}\left(b_{\lambda, \mu}(x) T_{m, j}-T_{m, j} b_{\lambda, \mu}(x)\right) \\
& \cdot D^{\gamma} T_{\lambda, \mu} f=\sum_{m=1}^{\infty} \sum_{j=1}^{d_{m}} \sum_{\lambda=1}^{\infty} \sum_{\mu=1}^{d_{\lambda}} a_{m, j}\left[b_{\lambda, \mu}, T_{m, j}\right] D^{\gamma} T_{\lambda, \mu} f .
\end{aligned}
$$

Therefore, together with (55), (60), and Lemma 11, we obtain

$$
\begin{aligned}
& \left\|\left(T_{1} \circ T_{2}-T_{1} T_{2}\right) D^{\gamma} f\right\|_{W M \dot{K}_{p, 1}^{\alpha, \lambda}\left(\mathbb{R}^{n}\right)} \\
& \leq \sum_{m=1}^{\infty} \sum_{j=1}^{d_{m}} \sum_{\lambda=1}^{\infty} \sum_{\mu=1}^{d_{\lambda}}\left\|a_{m, j}\right\|_{L^{\infty}} \\
& \cdot\left\|\left[b_{\lambda, \mu}, T_{m, j}\right] D^{\gamma} T_{\lambda, \mu} f\right\|_{W M \dot{K}_{p, 1}^{\alpha, \lambda}\left(\mathbb{R}^{n}\right)} \\
& \leq \sum_{m=1}^{\infty} \sum_{j=1}^{d_{m}} \sum_{\lambda=1}^{\infty} \sum_{\mu=1}^{d_{\lambda}}\left\|a_{m, j}\right\|_{L^{\infty}}\left\|D^{\gamma} b_{\lambda, \mu}\right\|_{\mathrm{BMO}} \\
& \cdot m^{n / 2+\gamma}\left\|T_{\lambda, \mu} f\right\|_{W M \dot{K}_{p, 1}^{\alpha, \lambda}\left(\mathbb{R}^{n}\right)} \\
& \leq \sum_{m=1}^{\infty} \sum_{j=1}^{d_{m}} \sum_{\lambda=1}^{\infty} \sum_{\mu=1}^{d_{\lambda}}\left\|a_{m, j}\right\|_{L^{\infty}}\left\|D^{\gamma} b_{\lambda, \mu}\right\|_{L^{\infty}} \\
& \cdot m^{n / 2+\gamma} \lambda^{n / 2}\|f\|_{M \dot{K}_{p, 1}^{\alpha, \lambda}\left(\mathbb{R}^{n}\right)} \\
& \leq \sum_{m=1}^{\infty} m^{n-2} m^{-2 n} m^{n / 2+\gamma} \sum_{\lambda=1}^{\infty} \lambda^{n-2} \lambda^{-2 n} \lambda^{n / 2}\|f\|_{M \dot{K}_{p, 1}^{\alpha, \lambda}\left(\mathbb{R}^{n}\right)} \\
& \leq\|f\|_{M \dot{K}_{p, 1}^{\alpha, \lambda}\left(\mathbb{R}^{n}\right)} .
\end{aligned}
$$

This finishes the proof of Theorem 2.

Proof of Theorem 3. We can estimate term (1) exactly as we did for the corresponding boundedness in Theorem 1 in the above arguments. Thus, we have only to prove (2) and (3) of Theorem 3. In order to do this, we use the same notations as in the proof of Theorem 2. By using the fact that $\Omega_{1}(x, y)$ and $\Omega_{2}(x, y)$ satisfy (10), therefore, we have

$$
\begin{aligned}
\left\|a_{m, j}\right\|_{L^{\infty}} & \leq m^{-2 n}, \\
\left\|b_{\lambda, \mu}\right\|_{L^{\infty}} & \leqslant \lambda^{-2 n} .
\end{aligned}
$$

Firstly, let us prove (2). As in the proof of Theorem 1, we can get

$$
\left(T_{1}^{\sharp}-T_{1}^{*}\right) \mathscr{I} f=\sum_{m=1}^{\infty} \sum_{j=1}^{d_{m}}(-1)^{m}\left[\bar{a}_{m, j}, T_{m, j}\right] \mathscr{I} f .
$$

As $\left[b, T_{m, j}\right]$ is a special Calderón-Zygmund operator, it is bounded from Morrey-Herz spaces $M \dot{K}_{p, 1}^{\alpha, \lambda}\left(\mathbb{R}^{n}\right)$ to weak Morrey-Herz spaces $W M \dot{K}_{p, 1}^{\alpha, \lambda}\left(\mathbb{R}^{n}\right)$ by applying Lemma 10 . Thus we have

$$
\left\|\left[b, T_{m, j}\right] f\right\|_{W M \dot{K}_{p, 1}^{\alpha, \lambda}\left(\mathbb{R}^{n}\right)} \leqslant m^{n / 2}\|b\|_{L^{\infty}}\|f\|_{M \dot{K}_{p, 1}^{\alpha, \lambda}\left(\mathbb{R}^{n}\right)} .
$$

Then by (65), we get

$$
\begin{aligned}
& \left\|\left(T_{1}^{\sharp}-T_{1}^{*}\right) \mathscr{I} f\right\|_{W M \dot{K}_{p, 1}^{\alpha, \lambda}\left(\mathbb{R}^{n}\right)} \\
& \quad \leqslant \sum_{m=1}^{\infty} m^{n-2} m^{-3 n / 2}\|f\|_{M \dot{K}_{p, 1}^{\alpha, \lambda}\left(\mathbb{R}^{n}\right)} \leqslant\|f\|_{M \dot{K}_{p, 1}^{\alpha, \lambda}\left(\mathbb{R}^{n}\right)} .
\end{aligned}
$$

Thus conclusion (2) is proved. 
We now estimate (3). Write

$$
\begin{aligned}
& \left(T_{1} \circ T_{2}-T_{1} T_{2}\right) \mathscr{I} f \\
& \quad=\sum_{m=1}^{\infty} \sum_{j=1}^{d_{m}} \sum_{\lambda=1}^{\infty} \sum_{\mu=1}^{d_{\lambda}}\left[b_{\lambda, \mu}, T_{m, j}\right] T_{\lambda, \mu} \mathscr{I} f .
\end{aligned}
$$

Therefore, by (65), (67), and Lemma 11, we get

$$
\begin{aligned}
& \left\|\left(T_{1} \circ T_{2}-T_{1} T_{2}\right) \mathscr{I} f\right\|_{W M \dot{K}_{p, 1}^{\alpha, \lambda}\left(\mathbb{R}^{n}\right)} \\
& \lesssim \sum_{m=1}^{\infty} \sum_{j=1}^{d_{m}} \sum_{\lambda=1}^{\infty} \sum_{\mu=1}^{d_{\lambda}}\left\|a_{m, j}\right\|_{L^{\infty}}\left\|b_{\lambda, \mu}\right\|_{L^{\infty}} \\
& \cdot m^{n / 2}\left\|T_{\lambda, \mu} \mathscr{I} f\right\|_{W M \dot{K}_{p, 1}^{\alpha, \lambda}\left(\mathbb{R}^{n}\right)} \lesssim \sum_{m=1}^{\infty} m^{n-2} \\
& \cdot m^{-2 n} m^{n / 2} \sum_{\lambda=1}^{\infty} \lambda^{n-2} \lambda^{-2 n} \lambda^{n / 2}\|f\|_{M \dot{K}_{p, 1}^{\alpha, \lambda}\left(\mathbb{R}^{n}\right)} \\
& \lesssim\|f\|_{M \dot{K}_{p, 1}^{\alpha, \lambda}\left(\mathbb{R}^{n}\right)} \cdot
\end{aligned}
$$

Thus conclusion (3) is also proved. Hence the proof of Theorem 3 is finished.

Proof of Theorem 4. In the first place, we will prove conclusion (1). Write $D=\sum_{k=1}^{n} \mathscr{R}_{k}\left(\partial / \partial x_{k}\right)$, where $\mathscr{R}_{k}$ denotes the Riesz transform. As in the proof of Theorem 1, we have

$$
\begin{aligned}
& (T D-D T) f(x)=\sum_{m=1}^{\infty} \sum_{j=1}^{d_{m}}\left[a_{m, j}, D\right] T_{m, j} f(x) \\
& =\sum_{m=1}^{\infty} \sum_{j=1}^{d_{m}} \sum_{k=1}^{n}\left[a_{m, j}, \mathscr{R}_{k} \frac{\partial}{\partial x_{k}}\right] T_{m, j} f(x) \\
& =\sum_{m=1}^{\infty} \sum_{j=1}^{d_{m}} \sum_{k=1}^{n} \mathscr{R}_{k}\left[a_{m, j}, \frac{\partial}{\partial x_{k}}\right] T_{m, j} f(x) \\
& \quad+\sum_{m=1}^{\infty} \sum_{j=1}^{d_{m}} \sum_{k=1}^{n}\left[a_{m, j}, \mathscr{R}_{k}\right] \frac{\partial}{\partial x_{k}}\left(T_{m, j} f\right)(x) \\
& =: I_{1}+I_{2} .
\end{aligned}
$$

We have by Leibniz's rules that

$$
I_{1}=\sum_{m=1}^{\infty} \sum_{j=1}^{d_{m}} \sum_{k=1}^{n} \mathscr{R}_{k}\left(\frac{\partial}{\partial x_{k}}\left(a_{m, j}\right) T_{m, j} f\right) .
$$

Thus we deduce from (43) that

$$
\begin{aligned}
& \frac{a_{m, j}}{\partial x_{k}}(x)=(-1)^{n} m^{-n}(m+n-2)^{-n} \\
& \quad \cdot \int_{S^{n-1}} \partial x_{k} L_{y^{\prime}}^{n}\left(\Omega\left(x, y^{\prime}\right)\right) Y_{m, j}\left(y^{\prime}\right) \mathrm{d} \sigma\left(y^{\prime}\right),
\end{aligned}
$$

From this and (12), we get for $k=1, \ldots, n$,

$$
\left\|\frac{\partial a_{m, j}}{\partial x_{k}}\right\|_{L^{\infty}} \lesssim m^{-2 n}
$$

By using the fact that $\left\|\mathscr{R}_{k} g\right\|_{W M \dot{K}_{p, 1}^{\alpha, \lambda}\left(\mathbb{R}^{n}\right)} \lesssim\|g\|_{M \dot{K}_{p, 1}^{\alpha, \lambda}\left(\mathbb{R}^{n}\right)}, d_{m} \simeq$ $m^{n-2}$, and Lemma 11, then we have

$$
\begin{aligned}
& \left\|I_{1}\right\|_{W M \dot{K}_{p, 1}^{\alpha, \lambda}\left(\mathbb{R}^{n}\right)} \\
& \quad \leq \sum_{m=1}^{\infty} \sum_{j=1}^{d_{m}} \sum_{k=1}^{n}\left\|\mathscr{R}_{k}\left(\frac{\partial}{\partial x_{k}}\left(a_{m, j}\right) T_{m, j} f\right)\right\|_{W M \dot{K}_{p, 1}^{\alpha, \lambda}\left(\mathbb{R}^{n}\right)} \\
& \quad \leq \sum_{m=1}^{\infty} \sum_{j=1}^{d_{m}} m^{-2 n} m^{n / 2}\|f\|_{M \dot{K}_{p, 1}^{\alpha, \lambda}\left(\mathbb{R}^{n}\right)} \\
& \quad \leq \sum_{m=1}^{\infty} m^{n-2} m^{-2 n} m^{n / 2}\|f\|_{M \dot{K}_{p, 1}^{\alpha, \lambda}\left(\mathbb{R}^{n}\right)} \leq\|f\|_{M \dot{K}_{p, 1}^{\alpha, \lambda}\left(\mathbb{R}^{n}\right)} .
\end{aligned}
$$

By Lemma 13 and (74), a trivial computation shows that, for $I_{2}$,

$$
\begin{aligned}
& \left\|I_{2}\right\|_{W M \dot{K}_{p, 1}^{\alpha, \lambda}\left(\mathbb{R}^{n}\right)} \\
& \quad \lesssim \sum_{m=1}^{\infty} \sum_{j=1}^{d_{m}} \sum_{k=1}^{n}\left\|\nabla a_{m, j}\right\|_{L^{\infty}}\left\|T_{m, j} f\right\|_{W M \dot{K}_{p, 1}^{\alpha, \lambda}\left(\mathbb{R}^{n}\right)} \\
& \quad \lesssim \sum_{m=1}^{\infty} \sum_{j=1}^{d_{m}} m^{-2 n} m^{n / 2}\|f\|_{M \dot{K}_{p, 1}^{\alpha, \lambda}\left(\mathbb{R}^{n}\right)} \\
& \quad \lesssim \sum_{m=1}^{\infty} m^{n-2} m^{-2 n} m^{n / 2}\|f\|_{M \dot{K}_{p, 1}^{\alpha, \lambda}\left(\mathbb{R}^{n}\right)} \lesssim\|f\|_{M \dot{K}_{p, 1}^{\alpha, \lambda}\left(\mathbb{R}^{n}\right)} .
\end{aligned}
$$

Combining the estimates above, we arrive at the desired boundedness

$$
\|(T D-D T) f\|_{W M \dot{K}_{p, 1}^{\alpha, \lambda}\left(\mathbb{R}^{n}\right)} \lesssim\|f\|_{M \dot{K}_{p, 1}^{\alpha, \lambda}\left(\mathbb{R}^{n}\right)} .
$$

We posterior prove conclusion (2). Write $D=\sum_{k=1}^{n} \mathscr{R}_{k}(\partial /$ $\left.\partial x_{k}\right)$; we have

$$
\begin{gathered}
\left(T^{\sharp}-T^{*}\right) D f(x)=\sum_{m=1}^{\infty} \sum_{j=1}^{d_{m}}(-1)^{m}\left[\bar{a}_{m, j}, T_{m, j}\right] D f(x) \\
=\sum_{k=1}^{n} \sum_{m=1}^{\infty} \sum_{j=1}^{d_{m}}(-1)^{m}\left[\bar{a}_{m, j}, T_{m, j}\right] \frac{\partial}{\partial x_{k}}\left(\mathscr{R}_{k} f\right)(x) .
\end{gathered}
$$

We now turn to estimate the $W M \dot{K}_{p, 1}^{\alpha, \lambda}\left(\mathbb{R}^{n}\right)$ norm of $\left[\bar{a}_{m, j}, T_{m, j}\right]\left(\partial / \partial x_{k}\right)\left(\mathscr{R}_{k} f\right)$. Applying (74), Lemma 13, and the fact that for any multi-index $\beta$ and $x \in \mathbb{R}^{n} \backslash\{0\}, m=1,2, \ldots$ (see [7]),

$$
\left|\partial^{\beta}\left(|x|^{m}\right) Y_{m, j}\right| \leq C(n)|x|^{m-|\beta|} m^{|\beta|+(n-2) / 2} .
$$


Hence, we get

$$
\begin{aligned}
& \left\|\left[\bar{a}_{m, j}, T_{m, j}\right] \frac{\partial}{\partial x_{k}}\left(\mathscr{R}_{k} f\right)\right\|_{W M \dot{K}_{p, 1}^{\alpha, \lambda}\left(\mathbb{R}^{n}\right)} \\
& \leq\left\|\nabla \bar{a}_{m, j}\right\|_{L^{\infty}} \max _{|\beta| \leq 2}\left\|\partial^{\beta} Y_{m, j}\right\|_{L^{\infty}\left(S^{n-1}\right)}\left\|\mathscr{R}_{k} f\right\|_{W M \dot{K}_{p, 1}^{\alpha, \lambda}\left(\mathbb{R}^{n}\right)} \\
& \leq m^{-2 n} m^{n / 2+1}\|f\|_{M \dot{K}_{p, 1}^{\alpha, \lambda}\left(\mathbb{R}^{n}\right)} \\
& \leq m^{-3 n / 2+1}\|f\|_{M \dot{K}_{p, 1}^{\alpha, \lambda}\left(\mathbb{R}^{n}\right)} .
\end{aligned}
$$

Combining the estimates of (78) with (80), we have

$$
\begin{aligned}
& \left\|\left(T^{\sharp}-T^{*}\right) D f\right\|_{W M \dot{K}_{p, 1}^{\alpha, \lambda}\left(\mathbb{R}^{n}\right)} \\
& \quad \lesssim \sum_{m=1}^{\infty} m^{n-2} m^{-3 n / 2+1}\|f\|_{M \dot{K}_{p, 1}^{\alpha, \lambda}\left(\mathbb{R}^{n}\right)} \lesssim\|f\|_{M \dot{K}_{p, 1}^{\alpha, \lambda}\left(\mathbb{R}^{n}\right)} .
\end{aligned}
$$

Consequently, the proof of Theorem 4 is completed.

Proof of Theorem 5. Similar to the proof of Theorem 2, we easily see that

$$
\begin{aligned}
& \left(T_{1} \circ T_{2}-T_{1} T_{2}\right) D f \\
& =\sum_{m=1}^{\infty} \sum_{d=1}^{d_{m}} \sum_{\lambda=1}^{\infty} \sum_{\mu=1}^{d_{\lambda}} a_{m, j}\left[b_{\lambda, \mu}, T_{m, j}\right] D T_{\lambda, \mu} f,
\end{aligned}
$$

where $a_{m, j}$ and $b_{\lambda, \mu}$ are same to occur in the proof of Theorem 2. By (10) and (12), we have

$$
\begin{aligned}
\left\|a_{m, j}\right\|_{L^{\infty}} & \leqslant m^{-2 n}, \\
\left\|\nabla b_{\lambda, \mu}\right\|_{L^{\infty}} & \leqslant \lambda^{-2 n} .
\end{aligned}
$$

Write $D=\sum_{k=1}^{n}\left(\partial / \partial x_{k}\right) \mathscr{R}_{k}$, and it then follows that

$$
\begin{aligned}
& \left\|\left(T_{1} \circ T_{2}-T_{1} T_{2}\right) D f\right\|_{W M \dot{K}_{p, 1}^{\alpha, \lambda}\left(\mathbb{R}^{n}\right)} \\
& \leq \sum_{m=1}^{\infty} \sum_{j=1}^{d_{m}} \sum_{\lambda=1}^{\infty} \sum_{\mu=1}^{d_{\lambda}}\left\|a_{m, j}\right\|_{L^{\infty}} \\
& .\left\|\left[b_{\lambda, \mu}, T_{m, j}\right]\left(\sum_{k=1}^{n} \frac{\partial}{\partial x_{k}} \mathscr{R}_{k} T_{\lambda, \mu} f\right)\right\|_{W M \dot{K}_{p, 1}^{\alpha, \lambda}\left(\mathbb{R}^{n}\right)} \\
& \leq \sum_{k=1}^{n} \sum_{m=1}^{\infty} \sum_{j=1}^{d_{m}} \sum_{\lambda=1}^{\infty} \sum_{\mu=1}^{d_{\lambda}}\left\|a_{m, j}\right\|_{L^{\infty}} \\
& .\left\|\left[b_{\lambda, \mu}, T_{m, j}\right]\left(\frac{\partial}{\partial x_{k}} \mathscr{R}_{k} T_{\lambda, \mu} f\right)\right\|_{M \dot{K}_{p, 1}^{\alpha, \lambda}\left(\mathbb{R}^{n}\right)} .
\end{aligned}
$$

The above estimates, via Lemma 13, lead to that

$$
\begin{aligned}
& \left\|\left(T_{1} \circ T_{2}-T_{1} T_{2}\right) D f\right\|_{W M \dot{K}_{p, 1}^{\alpha, \lambda}\left(\mathbb{R}^{n}\right)} \\
& \leq \sum_{m=1}^{\infty} \sum_{j=1}^{d_{m}} \sum_{\lambda=1}^{\infty} \sum_{\mu=1}^{d_{\lambda}}\left\|a_{m, j}\right\|_{L^{\infty}}\left\|\nabla b_{\lambda, \mu}\right\|_{L^{\infty}} \\
& \cdot \max _{|\beta| \leq 2}\left\|\partial^{\beta} Y_{m, j}\right\|_{L^{\infty}\left(S^{n-1}\right)}\left\|T_{\lambda, \mu} \mathscr{R}_{k} f\right\|_{W M \dot{K}_{p, 1}^{\alpha, \lambda}\left(\mathbb{R}^{n}\right)} .
\end{aligned}
$$

We thus obtain from (79), (83), and Lemma 11 that

$$
\begin{aligned}
& \left\|\left(T_{1} \circ T_{2}-T_{1} T_{2}\right) D f\right\|_{W M \dot{K}_{p, 1}^{\alpha, \lambda}\left(\mathbb{R}^{n}\right)} \\
& \lesssim \sum_{m=1}^{\infty} m^{n / 2-1} m^{-2 n} m^{n / 2+1} \sum_{\lambda=1}^{\infty} \lambda^{n / 2-1} \lambda^{-2 n} \lambda^{n / 2} \\
& \cdot\|f\|_{M \dot{K}_{p, 1}^{\alpha, \lambda}\left(\mathbb{R}^{n}\right)} \leqslant\|f\|_{M \dot{K}_{p, 1}^{\alpha, \lambda}\left(\mathbb{R}^{n}\right)} .
\end{aligned}
$$

Consequently, the proof of Theorem 5 is finished.

\section{Conflicts of Interest}

The authors declare that there are no conflicts of interest regarding the publication of this paper.

\section{Authors' Contributions}

All authors contributed equally to the writing of this paper. All authors read and approved the final manuscript.

\section{Acknowledgments}

This work is supported by National Natural Science Foundation of China (Grant no. 1156 1062).

\section{References}

[1] G. Di Fazio, D. K. Palagachev, and M. . Ragusa, "Global Morrey regularity of strong solutions to the Dirichlet problem for elliptic equations with discontinuous coefficients," Journal of Functional Analysis, vol. 166, no. 2, pp. 179-196, 1999.

[2] L. Diening and M. Ružička, "Calderón-Zygmund operators on generalized Lebesgue spaces Lp and problem related to fluid dynamics," Journal für die reine und Angewandte Mathematik, vol. 2003, no. 563, pp. 197-220, 2003.

[3] M. Christ, J. Duoandikoetxea, and J. L. Rubio de Francia, "Maximal operators related to the radon transform and the calderón-Zygmund method of rotations," Duke Mathematical Journal, vol. 53, no. 1, pp. 189-209, 1986.

[4] A. P. Calderón and A. Zygmund, "On singular integrals," American Journal of Mathematics, vol. 78, pp. 289-309, 1956.

[5] A. P. Calderón and A. Zygmund, "On Singular Integrals with Variable Kernels," Applicable Analysis: An International Journal, vol. 7, no. 3, pp. 221-238, 1978.

[6] S. P. Tao and Y. Q. Yang, "Boundedness of weighted MorreyHerz spaces with variable kernel singular integrals and fractional differentiations," Journal of Jilin University. Science Edition. Jilin Daxue Xuebao. Lixue Ban, vol. 54, no. 4, pp. 677-684, 2016.

[7] A. Calderón and A. Zygmund, "Singular integral operators and differential equations," American Journal of Mathematics, vol. 79, pp. 901-921, 1957.

[8] R. S. Strichartz, "Bounded mean oscillation and Sobolev spaces," Indiana University Mathematics Journal, vol. 29, no. 4, pp. 539-558, 1980.

[9] A. P. Calderón and A. Zygmund, "On a problem of Mihlin," Transactions of the American Mathematical Society, vol. 78, pp. 209-224, 1955. 
[10] Y. Chen and K. Zhu, "Weighted norm inequality for the singular integral with variable kernel and fractional differentiation," Journal of Mathematical Analysis and Applications, vol. 423, no. 2, pp. 1610-1629, 2015.

[11] J. Morrey, "On the solutions of quasi-linear elliptic partial differential equations," Transactions of the American Mathematical Society, vol. 43, no. 1, pp. 126-166, 1938.

[12] H. Triebel, "Local function spaces, Heat and Navier-Stokes Equations," EMS Tracts in Mathematics (ETM), vol. 20, 2013.

[13] S. Campanato, "Proprieties di una famiglia di spazi funzioni," Annali della Scuola Normale Superiore di Pisa, vol. 18, pp. 137160, 1964.

[14] D. R. Adams, Morrey Spaces, Lecture Notes in Applied and Numerical Harmonic Analysis, Birkhuser, 2015.

[15] D. R. Adams and J. Xiao, "Morrey spaces in harmonic analysis," Arkiv för Matematik, vol. 50, no. 2, pp. 201-230, 2012.

[16] J. A. Brudnyi, "Spaces defined by local approximations," Transactions of the Moscow Mathematical Society, vol. 24, pp. 69-132, 1971.

[17] M. Giaquinta, Multiple Integrals in the Calculus of Variations and Nonlinear Elliptic Systems, vol. 105 of Annals of Mathematics Studies, Princeton University Press, Princeton, NJ, USA, 1983.

[18] J. Peetre, "On the theory of Lp, $\lambda$ spaces," Journal of Functional Analysis, vol. 4, no. 1, pp. 71-87, 1969.

[19] L. Pick, A. Kufner, O. John, and S. Fučík, Function Spaces, vol. 1 of De Gruyter Series in Nonlinear Analysis and Applications 14, Berlin, 2nd edition, 2013.

[20] H. Rafeiro, N. Samko, and S. Samko, "Morrey-Campanato spaces: an overview," in Operator Theory, Pseudo-Differential Equations, and Mathematical Physics, vol. 228 of Operator Theory: Advances and Applications, pp. 293-324, Birkhuser, Basel, Switzerland, 2013.

[21] Y. Sawano, H. Gunawan, V. Guliyev, and H. Tanaka, "Morrey spaces and related function spaces," Journal of Function Spaces, vol. 2014, Article ID 867192, 2014.

[22] A. Beurling, "Construction and analysis of some convolution algebras," Annales de l'Institut Fourier, vol. 14, pp. 1-32, 1964.

[23] C. S. Herz, "Lipschitz spaces and Bernstein's theorem on absolutely convergent Fourier transforms," Journal of Mathematics and Mechanics, vol. 18, pp. 283-324, 1968.

[24] I. Baernstein and E. T. Sawyer, "Embedding and multiplier theorems for $\mathrm{Hp}(\mathrm{Rn})$," Memoirs of the AMS - American Mathematical Society, vol. 53, no. 318, iv+82 pages, 1985.

[25] S. Lu and D. Yang, "Herz-type Sobolev and Bessel potential spaces and their applications," Science China Mathematics, vol. 40, no. 2, pp. 113-129, 1997.

[26] M.-Y. Lee, "Convolution operators on the weighted Herz-type HARdy spaces," Journal of Approximation Theory, vol. 138, no. 2, pp. 197-210, 2006.

[27] S. Z. Lu and D. C. Yang, "The decomposition of the weighted Herz spaces and its applications," Science in China. Series A, Mathematics, physics, astronomy, vol. 38, no. 2, pp. 147-158, 1995.

[28] S. Lu, D. Yang, and G. Hu, Herz type spaces and their applications, Science Press, Beijing, China, 2008.

[29] S. $\mathrm{Lu}$ and $\mathrm{L} . \mathrm{Xu}$, "Boundedness of rough singular integral operators on the homogeneous Morrey-Herz spaces," Hokkaido Mathematical Journal, vol. 34, no. 2, pp. 299-314, 2005.

[30] S. P. Tao and W. Cao, "Boundedness of commutators for a class of sublinear operators in weak Morrey-Herz spaces on homogeneous spaces," Journal of Mathematics, vol. 31, no. 1, pp. 115-122, 2011.
[31] S. P. Tao and X. K. Shao, "Boundedness of Marcinkiewicz integrals with variable kernel on homogeneous MORreyHerz spaces," Journal of Lanzhou University. Natural Sciences. Lanzhou Daxue Xuebao. Ziran Kexue Ban, vol. 46, no. 3, pp. 102107, 2010.

[32] L. Wang, "Boundedness of fractional sub-linear operators and its commutators on weak Morrey-Herz spaces on homogeneous spaces," Journal of Math, vol. 36, no. 2, pp. 353-364, 2016.

[33] J. Duoandikoetxea, Fourier Analysis, American Mathematical Society, 2001.

[34] A. P. Calderón, "Commutators of singular integral operators," Proceedings of the National Acadamy of Sciences of the United States of America, vol. 53, pp. 1092-1099, 1965.

[35] M. A. Murray, "Commutators with fractional differentiation and BMO Sobolev spaces," Indiana University Mathematics Journal, vol. 34, no. 1, pp. 205-215, 1985.

[36] G. Di Fazio and M. A. Ragusa, "Interior estimates in Morrey spaces for strong solutions to nondivergence form equations with discontinuous coefficients," Journal of Functional Analysis, vol. 112, no. 2, pp. 241-256, 1993. 




Advances in

Operations Research

vatersals

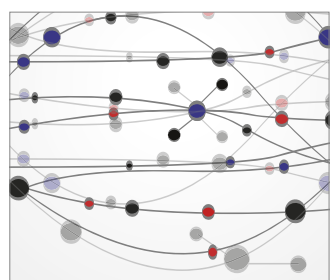

\section{The Scientific} World Journal
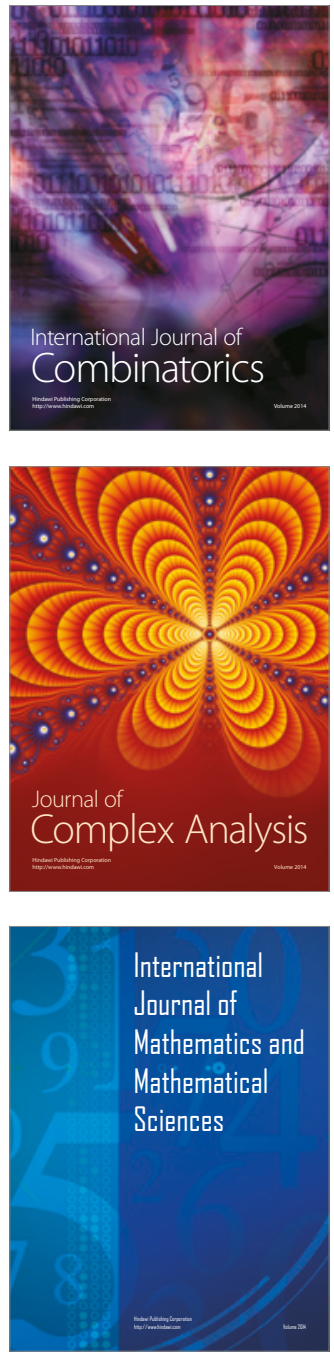


Algebra

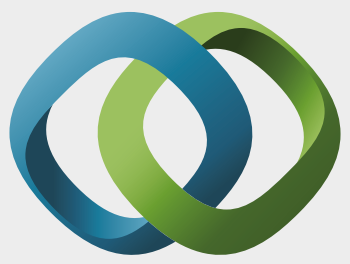

\section{Hindawi}

Submit your manuscripts at

https://www.hindawi.com
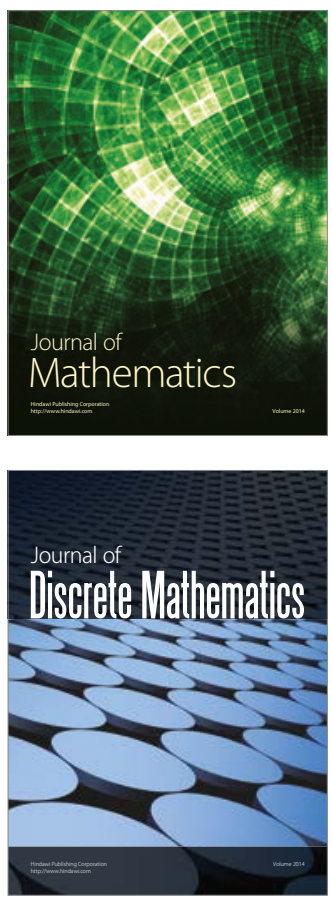



Mathematical Problems in Engineering
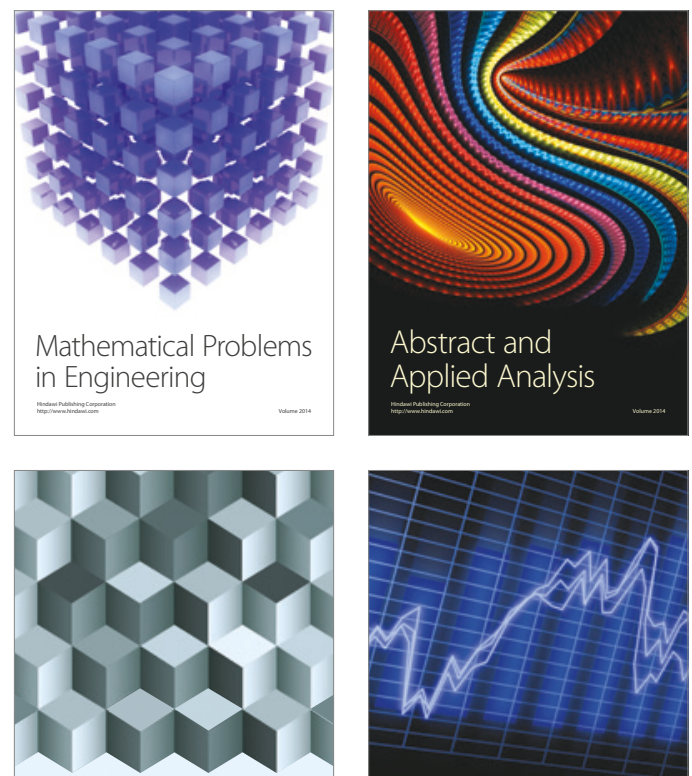

Journal of

Function Spaces

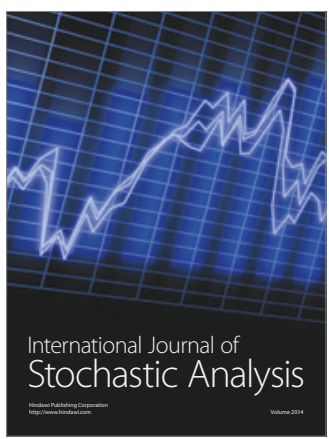

Probability and Statistics
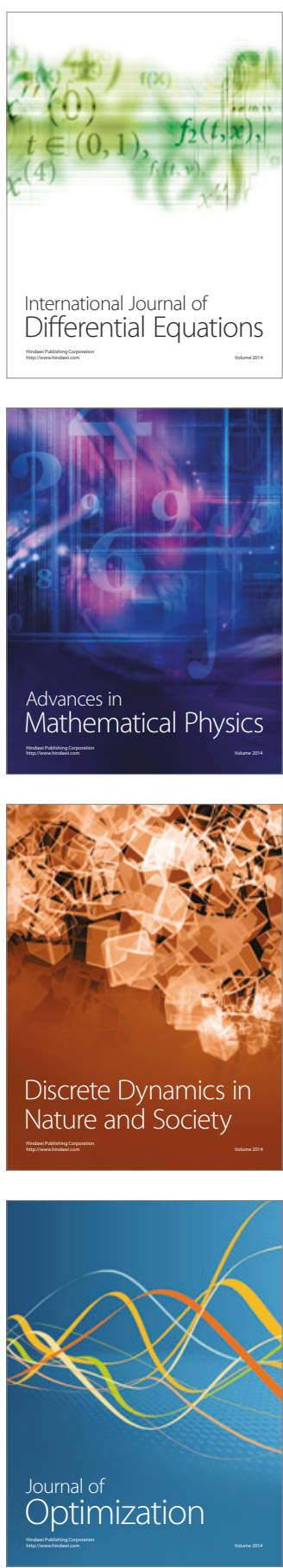